\title{
Combination aerosol therapy to treat Burkholderia cepacia complex
}

\author{
P.G. Middleton*, T.J. Kidd" and B. Williams"
}

ABSTRACT: Burkholderia cepacia infection in cystic fibrosis (CF) patients is associated with significant morbidity and mortality, yet no definitive treatment is currently available. This report describes a new approach to treat $B$. cepacia infection in CF patients, using a combination of amiloride and tobramycin aerosols.

Four adults with the typical clinical syndrome of CF were recruited after repeated positive sputum cultures for $B$. cepacia. Aerosols of amiloride and tobramycin were given three times daily for 1-6 months, and repeated sputum cultures were collected to assess efficacy.

Three of the four patients treated with the combined therapy eradicated $B$. cepacia from their sputum cultures for at least 2 yrs, and there were no adverse events.

This novel combination may provide a new therapeutic option for Burkholderia cepacia infections. Furthermore, the strategy of combining antibiotics with ion transport agents may have ramifications for the treatment of other multi-resistant organisms.

\section{KEYWORDS: Aerosol, amiloride, Burkholderia cepacia complex, cystic fibrosis, tobramycin}

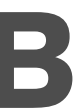

urkholderia (previously Pseudomonas) cepacia is an important pathogen in cystic fibrosis (CF) lung disease. B. cepacia infection/colonisation in CF patients was first recognised $30 \mathrm{yrs}$ ago, and the "cepacia syndrome" of severe, progressive, necrotising pneumonia leading to death from septicaemia/ respiratory failure was noted soon afterwards [1]. The spectrum of outcomes following $B$. cepacia colonisation varies from the "cepacia syndrome" through an accelerated decline in lung function to no apparent adverse effects; following transplantation, the presence of $B$. cepacia is associated with worse outcome, so $B$. cepacia colonisation is generally regarded as a relative or absolute contraindication to transplantation.

B. cepacia is often resistant to many antibiotics, displaying both intrinsic and inducible resistance, so antibiotic therapy is generally suppressive, rather than curative. Consequently, the mainstay of $B$. cepacia management in the $\mathrm{CF}$ clinic remains segregation and isolation of colonised patients to prevent spread of the organism. However, previous in vitro studies demonstrated that the sodium channel blocker amiloride can act synergistically with tobramycin against 50\% of B. cepacia isolates [2]. As this data pre-dated the determination of the different genomovars of $B$. cepacia complex, it is unclear whether the susceptibility of some organisms was related to inherent differences between genomovars or phenotypic differences within the same organism.

Although both nebulised amiloride [3, 4] and tobramycin [5] have been used separately in CF, therapeutic use of this combination has not previously been reported. The current study now presents a series of four CF patients with different genomovars of the $B$. cepacia complex who were treated with nebulised amiloride and tobramycin.

\section{METHODS}

All patients had typical CF syndromes and were diagnosed $<18$ months of age, with abnormal sweat tests; all were chronically colonised with Pseudomonas aeruginosa. Following three positive sputum cultures for B. cepacia, the patients were approached about the use of the combination therapy. Preservative free solutions of amiloride were produced under sterile conditions in the hospital pharmacy, with a standard single dose of $5 \mathrm{mLs}$ of $1 \mathrm{mM}$ solution dissolved in $0.45 \%$ saline given $t . d$.s, followed by tobramycin $(80 \mathrm{mg}$ in $2 \mathrm{~mL}$ ) t.d.s. The first doses were given under
AFFILIATIONS

*Ludwig Engel Centre for Respiratory Research, Dept of Respiratory Medicine, and

'Dept of Pharmacy, Westmead Hospital, Westmead NSW,

\#Dept of Microbiology, Queensland Health Pathology Service, The Prince Charles Hospital, Chermside Qld, Australia.

CORRESPONDENCE

P.G. Middleton

University of Sydney

CF Unit

Ludwig Engel Centre for Respiratory Research

Dept of Respiratory Medicine

Westmead Hospital

Westmead

2145, NSW

Australia

Fax: 61298457286

E-mail:

peterm@westgate.wh.usyd.edu.au

Received:

October 302004

Accepted after revision:

May 032005 
clinical supervision, though no adverse reactions were seen. During therapy with amiloride/tobramycin no adverse events were recorded, in particular there was no bronchoconstriction, nor changes in serum electrolytes. Use of this combined therapy was approved by the Drug Committee of Westmead Hospital (NSW, Australia) and all patients gave written informed consent.

\section{RESULTS \\ Case 1}

Following three consecutive positive sputum cultures for $B$. cepacia complex, this 23 -yr-old male $(\Delta \mathrm{F} 508 / \Delta \mathrm{F} 508)$ with stable lung function and clinical status commenced open label treatment with amiloride/tobramycin. Unfortunately genomovar testing was not available at this time. Following 1 month of therapy, sputum cultures were negative for B. cepacia complex at 1, 2, 4 and 6 months, and lung function/clinical status remained stable. However, at 7 months, culture revealed a further scanty growth of B. cepacia complex, so a further 6 months of treatment was given. Repeat sputum cultures during therapy $(1,2,3,13,26$ weeks) were all negative. Ten cultures over the 2 yrs since cessation of therapy remain negative for $B$. cepacia complex, although the $P$. aeruginosa remains.

\section{Case 2}

Following three consecutive sputum cultures positive for $B$. cepacia, this $21-\mathrm{yr}-\mathrm{old}$ male $(\Delta \mathrm{F} 508 / \Delta \mathrm{F} 508)$, also with stable lung function and clinical status, commenced nebulised amiloride/tobramycin therapy for 1 month. Sputum culture at the end of treatment (1 month) was negative, and five repeat sputum cultures over the 2 yrs following treatment have been negative for $B$. cepacia complex, although the $P$. aeruginosa remains. Subsequent genomovar testing of the original strain using standard methods [6-8] confirmed $B$. cepacia genomovar III recA clade $\mathrm{A}$, now designated Burkholderia cenocepacia.

\section{Case 3}

This 21-yr-old male (G551D/unknown) had relatively preserved lung function (forced expiratory volume in one second $\sim 75 \%$ predicted), but poor hepatic function with advanced cirrhosis and splenomegaly. Following the first scanty growth of $B$. cepacia complex, repeat cultures 4 and 6 weeks later showed heavy growth of $B$. cepacia complex, subsequently demonstrated to be Burkholderia ambifaria (Genomovar VII). As the patient had a clinical exacerbation at this time, standard intravenous anti-pseudomonal antibiotics were given as an inpatient, and the amiloride/tobramycin therapy was commenced. After 2, 3 and 4 weeks of treatment, sputum culture showed moderate, scanty and no growth of B. cepacia complex respectively. Amiloride/tobramycin treatment was continued for a total of 4 months. Over the subsequent 2 yrs, a total of 30 sputum samples have all been negative for B. ambifaria, as shown in figure 1. From a respiratory viewpoint the patient remained stable, but when his liver failure progressed, he was accepted for liver transplant; which was performed in 2004, just over 2 yrs since the last isolation of B. ambifaria. Following liver transplant, the B. ambifaria has still not been isolated, although the $P$. aeruginosa remains.

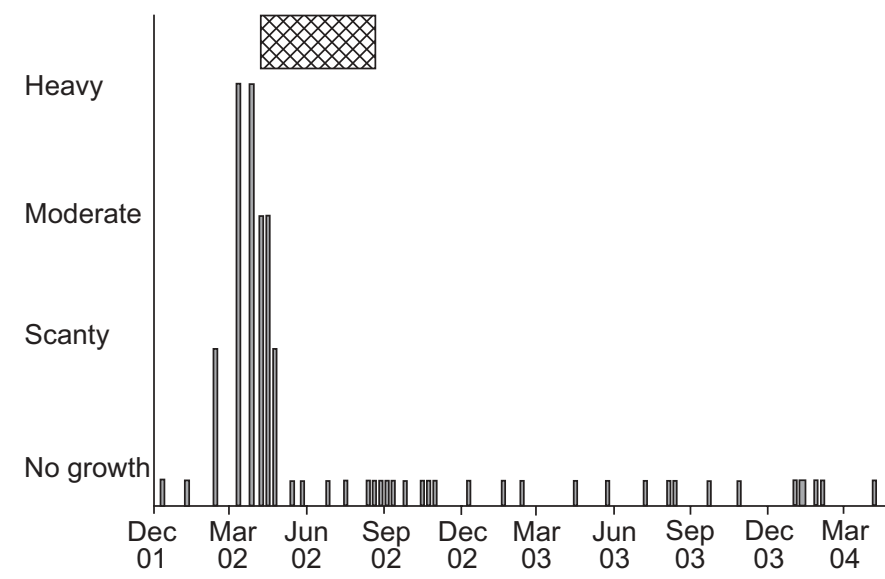

FIGURE 1. The time course of Burkholderia cepacia complex cultures for case 3. $\mathbf{D}$ : the period of treatment with amiloride/tobramycin

\section{Case 4}

This 26-yr-old female $(\Delta \mathrm{F} 508 / \Delta \mathrm{F} 508)$ had a single scanty growth of $B$. cepacia 18 months before presentation, with a further positive culture 3 months before presentation. On presentation, a moderate growth of $B$. cepacia was found, and subsequent genomovar testing showed $B$. cenocepacia recA clade $B$. Clinically she had a mild exacerbation at this time, and the chest radiograph showed a small area of collapse in the left upper lobe. Intravenous antibiotics, mucolytics, physiotherapy and amiloride/tobramycin aerosols were not successful in resolving the left upper lobe atelectasis or treating the $B$. cenocepacia. Despite 3 months of amiloride and tobramycin the B. cenocepacia continues to be isolated, and the patient remains stable clinically.

\section{DISCUSSION}

Following nebulised amiloride and tobramycin therapy, sputum cultures were negative for $B$. cepacia complex in three of the four CF patients treated. This suggests that prolonged amiloride/tobramycin aerosol therapy may suppress or potentially eradicate $B$. cepacia complex, at least in some instances. However, a number of important issues need to be addressed. Firstly, the definitions of colonisation and eradication are not agreed. The number of positive sputum cultures required to consider a patient colonised, and the number of subsequent negative sputum cultures required to categorise an organism as eradicated is yet to be determined. Until there is consensus, the natural history of B. cepacia colonisation/ infection following initial isolation will be difficult to ascertain. In a large retrospective study of approximately $450 \mathrm{CF}$ patients in British Columbia (Canada) over 17 yrs B. cepacia complex was isolated from 62 patients [9]. Using a definition of colonisation as one positive sputum culture and eradication as three consecutive negative cultures, nine of 46 infected with genomovar III were deemed "transient". More recently JONES et al. [10] performed a retrospective analysis of the Manchester Adult Cystic Fibrosis Centre (UK). Using definitions of one positive culture as colonisation and three consecutive negative cultures over at least 12 months as eradication, they reported frequent $(\sim 50 \%)$ spontaneous resolution of infection/colonisation with Burkholderia multivorans (genomovar II) without 
specific treatment. There were only rare cases of spontaneous resolution with $B$. cenocepacia (genomovar III), but no cases of infection with B. ambifaria were reported [10]. In the current study, slightly stricter definitions were used; colonisation as $\geqslant 3$ sputum positive cultures over at least 1 month and eradication as $\geqslant 5$ negative sputum cultures over at least 3 months. Using these definitions, three of the four patients in the current study eradicated the $B$. cepacia complex, but it should be noted that in this pilot study there was no control group, so it may be argued that the infection/colonisation could have resolved spontaneously.

It is possible that the negative sputum cultures in the current study represent suppression, rather than true eradication of the organism, only further repeated sputum sampling over the next few years will confirm eradication. LipumA et al. [11] have described a case where nine bimonthly sputum cultures were negative for $B$. cepacia complex before a positive culture at 24 months. In the current study, multiple sputum cultures in cases 1 and 2 have remained negative for at least 2 yrs following treatment. In case 3, 30 sputum cultures collected over 2 yrs have all remained negative and even with immunosuppression following liver transplantation, the $B$. ambifaria has still not been isolated. Finally, although the $B$. cepacia complex isolates in this study were resistant to tobramycin and other antibiotics (tested individually) the role of concurrent intravenous antibiotics given in case 3 against the B. ambifaria is not known.

The mechanism of in vivo synergy between amiloride and tobramycin against B. cepacia is not known. The initial work by COHN et al. [12] demonstrated synergy between amiloride and tobramycin in vitro, with greater effects from some amiloride analogues. More recently, different substituted amine compounds demonstrated synergy with tobramycin against a number of $B$. cepacia isolates [13]. This suggests that the amine group of amiloride is important in the observed synergy with tobramycin. CoHn et al. [14] have also demonstrated that high salt concentrations in the growth medium decreased the antibacterial effect of tobramycin against $B$. cepacia, an effect that was prevented by amiloride pre-treatment. Overall it would appear likely that the amiloride, perhaps through its amine moiety, directly alters the $B$. cepacia, interfering with the mechanism(s) used to protect the bacteria from aminoglycosides. Interestingly, CF sputum exhibits increased ammonium concentrations which decrease following antibiotic therapy [15], although whether this is involved in the amiloride and tobramycin synergy is unknown. The current authors have previously demonstrated a link between antibiotics and ion transport, as a different antibiotic, trimethoprim, blocks epithelial sodium transport [16], but the involvement of sodium transport in bacterial killing by tobramycin or by sulphur/trimethoprim combinations is not clear. As the antimicrobial activity of amiloride alone is very small [12], it is unlikely to be involved in the amiloride/tobramycin synergy observed in the current study.

An alternative explanation may be that the amiloride alters airway ion transport rather than bacterial ion transport. This may induce a subtle change in airway surface liquid, which improves the bactericidal effect of the tobramycin or potentially the innate pulmonary defence mechanisms. As the concentration of amiloride following nebulisation has been estimated to be $\sim 0.1 \mathrm{mM}$ [3], airway ion transport may have been altered in the current study, so further studies are necessary to determine the likely mechanism(s) of synergy between amiloride and tobramycin in vivo. Controlled trials of this novel therapeutic strategy are also required in a larger group of subjects, especially those with chronic Burkholderia cepacia complex infection.

In conclusion, this pilot study suggests that amiloride/ tobramycin aerosols may reduce the chronic infection rate following initial isolation of Burkholderia cepacia complex from the cystic fibrosis airway, and if confirmed will have important therapeutic ramifications for cystic fibrosis care. The combination of antibiotics and ion channel agents may also prove useful in the treatment of other multi-resistant bacteria.

\section{ACKNOWLEDGEMENTS}

The authors would like to thank the Drug Committee of Westmead Hospital (Westmead, NSW, Australia) for approving this nonstandard use of nebulised therapy and the patients for agreeing to be involved in this trial.

\section{REFERENCES}

1 Isles A, Maclusky I, Corey M, et al. Pseudomonas cepacia infection in cystic fibrosis: an emerging problem. J Pediatr 1984; 104: 206-210.

2 Cohn RC, Jacobs M, Aronoff SC. In vitro activity of amiloride combined with tobramycin against Pseudomonas isolates from patients with cystic fibrosis. Antimicrob Agents Chemother 1988; 32: 395-396.

3 Knowles MR, Church NL, Waltner WE, et al. A pilot study of aerosolized amiloride for the treatment of lung disease in cystic fibrosis. N Engl J Med 1990; 322: 1189-1194.

4 Graham A, Hasani A, Alton EWFW, et al. No added benefit from nebulized amiloride in patients with cystic fibrosis. Eur Respir J 1993; 6: 1243-1248.

5 Touw DJ, Brimicombe RW, Hodson ME, Heijerman HG, Bakker W. Inhalation of antibiotics in cystic fibrosis. Eur Respir J 1995; 8: 1594-1604.

6 Segonds C, Heulin T, Marty N, Chabanon G. Differentiation of Burkholderia species by PCR-restriction fragment length polymorphism analysis of the 16S rRNA gene and application to cystic fibrosis isolates. J Clin Microbiol 1999; 37: 2201-2208.

7 Mahenthiralingam E, Bischof J, Byrne SK, et al. DNA-Based diagnostic approaches for identification of Burkholderia cepacia complex, Burkholderia vietnamiensis, Burkholderia multivorans, Burkholderia stabilis, and Burkholderia cepacia genomovars I and III. J Clin Microbiol 2000; 38: 3165-3173.

8 Henry DA, Mahenthiralingam E, Vandamme P, Coenye T, Speert DP. Phenotypic methods for determining genomovar status of the Burkholderia cepacia complex. J Clin Microbiol 2001; 39: 1073-1078.

9 Mahenthiralingam E, Vandamme P, Campbell ME, et al. Infection with Burkholderia cepacia complex genomovars in patients with cystic fibrosis: virulent transmissible strains 
of genomovar III can replace Burkholderia multivorans. Clin Infect Dis 2001; 33: 1469-1475.

10 Jones AM, Dodd ME, Govan JRW, et al. Burkholderia cenocepacia and Burkholderia multivorans: influence on survival in cystic fibrosis. Thorax 2004; 59: 948-951.

11 Lipuma JJ, Marks-Austin KA, Holsclaw DS, Jr, Winnie GB, Gilligan PH, Stull TL. Inapparent transmission of Pseudomonas (Burkholderia) cepacia among patients with cystic fibrosis. Pediatr Infect Dis J 1994; 13: 716-719.

12 Cohn RC, Rudzienski L, Putnam RW. In vitro antimicrobial activity of amiloride analogs against Pseudomonas. Chemotherapy 1992; 38: 232-237.
13 Cohn RC, Rudzienski L. Substituted amines synergistic with tobramycin against Burkholderia cepacia in vitro. Chemotherapy 1997; 43: 100-107.

14 Cohn RC, Aronoff SC. The effect of sodium on amiloridetobramycin synergy in Pseudomonas cepacia. J Lab Clin Med 1989; 114: 724-727.

15 Gaston B, Ratjen F, Vaughan JW, et al. Nitrogen redox balance in the cystic fibrosis airway: effects of antipseudomonal therapy. Am J Respir Crit Care Med 2002; 165: 387-390.

16 Middleton PG, Geddes DM, Alton EWFW. Trimethoprim and tetracycline inhibit airway epithelial sodium absorption. Am J Respir Crit Care Med 1996; 154: 18-23. 